\title{
Генерация поверхностных электронных состояний на границе раздела кремний-сверхтонкий окисел в процессе полевого повреждения структур металл-окисел-полупроводник
}

\author{
(C) Е.И. Гольдман, С.А. Левашов, В.Г. Нарышкина, Г.В. Чучева \\ Фрязинский фрилиал Института радиотехники и электроники им. В.А. Котельникова Российской академии наук, \\ 141190 Фрязино, Россия \\ E-mail: gvc@ms.ire.rssi.ru
}

(Получена 10 января 2017 г. Принята к печати 20 января 2017 г.)

\begin{abstract}
Проведены измерения высокочастотных вольт-фарадных характеристик структур металл-окисел-полупроводник (МОП) на $n$-Si-MOП подложках с толщиной окисла $39 \AA \AA$, подвергнутых повреждению при полевом стрессе. Показано, что воздействие на сверхтонкий изолирующий слой сильного, но допробойного, электрического поля приводит к образованию большого числа дополнительных пограничных локализованных электронных состояний с уровнем, отстоящим от дна зоны проводимости кремния на 0.14 эВ. Обнаружено, что перезарядка вновь образованных центров с ростом полевого напряжения обеспечивает накопление у границы раздела кремний-окисел избыточного заряда до $8 \cdot 10^{12} \mathrm{~cm}^{-2}$. Время жизни, рожденных при полевом воздействии, локальных центров составляет двое суток; после этого зависимости заряда, локализованного на границе раздела полупроводник-диэлектрик, от напряжения на затворе в состояниях до стресса и после стресса практически совпадают.
\end{abstract}

DOI: 10.21883/FTP.2017.09.44881.8512

Воздействие сильного, но допробойного, электрического поля на слои окислов кремния приводит к так называемому „Повреждению“ изоляторов, выражающемуся в увеличении их туннельной проводимости, и возрастанию концентрации локализованных электронных состояний на границе раздела (ГР) полупроводник-диэлектрик [1]. Исследования этих явлений для достаточно толстых (более $5 \mathrm{Hм}$ ) слоев $\mathrm{SiO}_{2}$ проводятся уже более 40 лет [2], и к настоящему времени развиты достаточно полные представления о механизмах проявляющихся эффектов [3-6]. Разработка наномасштабных электронных устройств стимулировала изучение повреждения тонких, и в особенности сверхтонких (толщиной менее 4 нм), изолирующих пленок. Полученные в этой связи результаты в основном описывают увеличение туннельной проводимости окисла за счет накопления на ГР в процессе стресса дополнительного встроенного заряда [1,7]. Образование центров генерации дырок во время полевого повреждения кремниевых структур металл-окисел-полупроводник (МОП) с толщиной окисла $\sim 40 \AA$ рассматривалось в работе [8]. Собственно из анализа данных динамических вольт-амперных характеристик образца после стресса фиксировалось повышение темпа рождения неосновных носителей заряда в состоянии обеднения ГР. Непосредственную информацию об изгибе зон в полупроводнике, изменении заряда пограничных состояний и концентрации неосновных носителей заряда у ГР можно получать из вида высокочастотных емкостных характеристик структур металл-диэлектрик-полупроводник [9]. В данной работе будут описаны результаты, полученные на основе измерений высокочастотных емкостей $n$-Si-MOП структур в динамическом режиме.
Есть несколько особенностей исследований свойств ГР полупроводник-диэлектрик на основе полевых зависимостей высокочастотной емкости структур со сверхтонкими изолирующими слоями по сравнению со слоями относительно толстых объектов [10]. Во-первых, это необходимость учета возможного проявления вкладов проводимости полупроводниковой подложки, туннельных токов через окисел и процессов генерации неосновных носителей заряда. Во-вторых, необходимы проведение измерений с повышенной точностью и применение сглаживающих шумы методов обработки экспериментальных данных. Наши опыты проводились на $n$ - $\mathrm{Si}-\mathrm{MOП} \mathrm{структурах,} \mathrm{аналогичных} \mathrm{использованным}$ в [11]. Как показано в указанной работе, величина активной составляющей импеданса этих образцов лежит вне области допустимых измерений, а в емкостных характеристиках из особенностей для сверхтонких изоляторов проявляется только сопротивление полупроводниковой части. Это означает, что достаточно измерять полевые характеристики реактивной составляющей импеданса объектов только на двух высоких частотах и из них определять и проводимость подложки $[10,12]$, и емкость поверхностной заряженной области у ГР полупроводник-диэлектрик.

Эксперименты проводились на частотах 0.5 и 1 МГц с использованием прецизионного измерителя LCR Agilent E4980A на $n$-Si-MOП структурах с толщиной окисла $39 \AA$ и площадью полевого электрода $S=1.6 \cdot 10^{-3} \mathrm{~cm}^{2}$. Образцы подвергались полевому стрессу, так же как и в [8]: при комнатной температуре выдерживались в течение 1ч при напряжении на полевом электроде $V_{g}=-3.8 \mathrm{~B}$. Таким образом, при обедняющей полупроводник полярности $V_{g}$ учитывались задержка полевого воздействия на изолятор в связи с 
медленным накоплением у ГР неосновных носителей заряда (дырок) и частичное падение внешнего напряжения на кремнии в стационарном состоянии. Измерение импеданса структур проводилось в динамическом режиме, $V_{g}$ изменялось со временем с постоянной скоростью полевой развертки $\beta=15 \mathrm{MB} / \mathrm{c}$ вначале от $1.5 \mathrm{~B}$ до $-1.5 \mathrm{~B}$ (прямая ветвь), затем от $-1.5 \mathrm{~B}$ до $1.5 \mathrm{~B}$ (обратная ветвь). Такое ограничение амплитуды развертки полевого напряжения предохраняло образец от

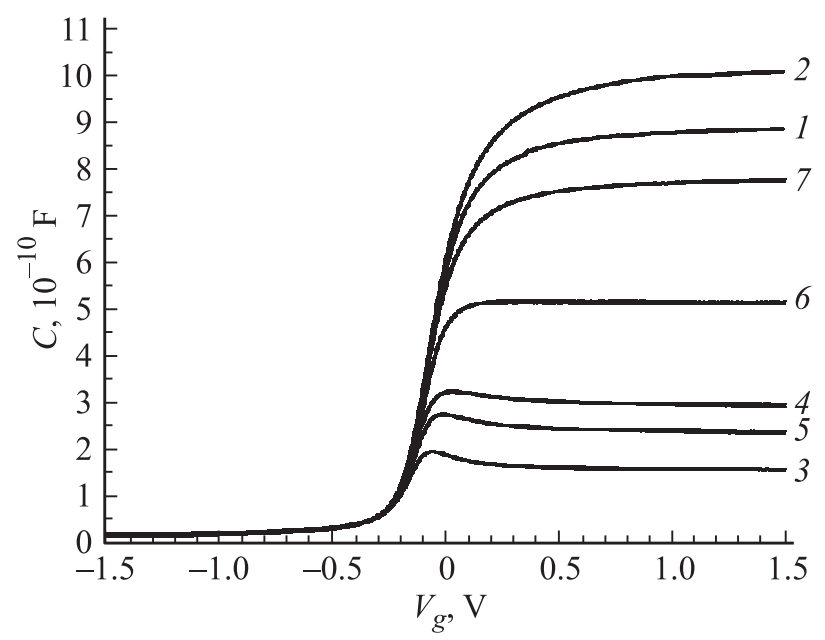

Рис. 1. Высокочастотные вольт-фарадные характеристики $\mathrm{Si}-\mathrm{MOП} \mathrm{структуры,} \mathrm{измеренные} \mathrm{до} \mathrm{и} \mathrm{после} \mathrm{полевого} \mathrm{воз-}$ действия. 1 - до стресса, 1 МГц; 2 - до стресса, 0.5 МГц; 3 - сразу после стресса, 1 МГц; 4 - сразу после стресса, 0.5 МГц; 5 - через 2 ч после стресса, 1 МГц; 6 - через 2 ч после стресса, $0.5 \mathrm{MГц;} 7$ - через 4 ч после стресса, 0.5 МГц. В масштабе рисунка кривые, соответствующие состоянию через 2 дня после стресса при 1 МГц и $0.5 \mathrm{MГц,}$ слились с линиями 1 и 2 соответственно. График, отвечающий измерениям через 4 ч после стресса при 1 МГц, практически совпал с кривой 6 .

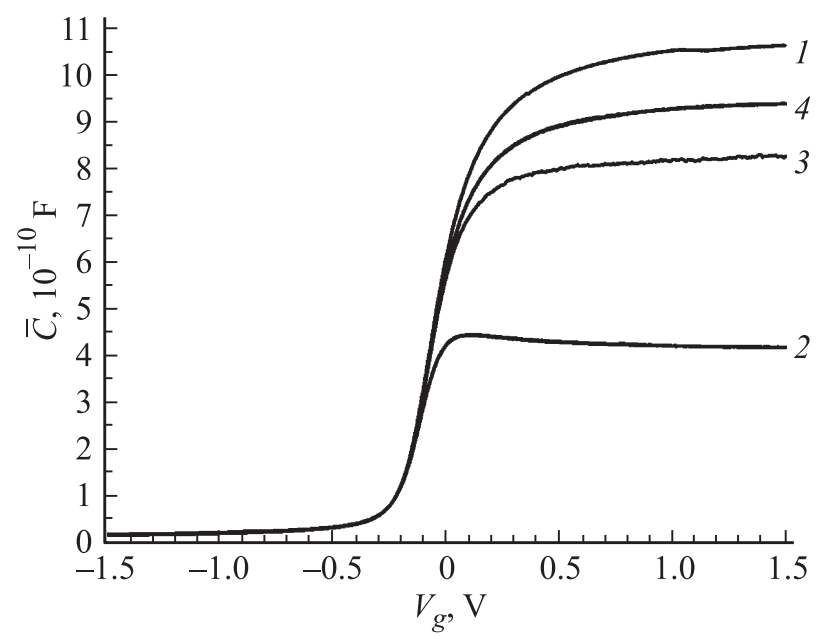

Рис. 2. Высокочастотные полевые зависимости емкости $\bar{C}\left(V_{g}\right)$ : 1 - до стресса, 2 - сразу после стресса, 3 - через 2 ч после стресса, 4 - через 4 ч после стресса. В масштабе рисунка кривая, соответствующая состоянию через 2 дня после стресса, слилась с линией 1 . дополнительного повреждения в процессе регистрации его емкости. На рис. 1 показаны вольт-фарадные характеристики образцов $C_{1}\left(V_{g}\right)-0.5 \mathrm{M} ц$ и $C_{2}\left(V_{g}\right)-1 \mathrm{M} ц$ на различных до стрессовых и после стрессовых стадиях. Мы полагаем, что для частот, на которых проводились измерения, перезарядка поверхностных состояний и генерация-рекомбинация неосновных носителей заряда не успевают откликаться на колебания внешнего напряжения. Высокочастотная емкость $\bar{C}$, отвечающая поверхностному заряженному слою полупроводника и изолирующему промежутку, а также проводимость полупроводниковой подложки $\rho_{b}$ определялись в соответствии с работами $[10,12]$ из экспериментальных значений $C_{1}\left(V_{g}\right)$ и $C_{2}\left(V_{g}\right)$ по формулам:

$$
\begin{gathered}
\bar{C}=\frac{C_{1} C_{2}\left(\omega_{1}^{2}-\omega_{2}^{2}\right)}{\omega_{1}^{2} C_{1}-\omega_{2}^{2} C_{2}}, \\
\rho_{b}=\frac{\sqrt{\left(C_{2}-C_{1}\right)\left(\omega_{1}^{2} C_{1}-\omega_{2}^{2} C_{2}\right)}}{C_{1} C_{2}\left(\omega_{1}^{2}-\omega_{2}^{2}\right)},
\end{gathered}
$$

где $\omega_{1}$ и $\omega_{2}$ - циклические частоты изменения тестирующего напряжения.

Графики $\bar{C}\left(V_{g}\right)$ представлены на рис. 2, кривые прямой и обратной ветви практически совпадают, они различимы только в области генерации дырок, которая запаздывает и проявляется в основном на обратной ветви. Вычисленная зависимость $\rho_{b}\left(V_{g}\right)$ имеет вид плато со значением $\rho_{b} \approx 65 \mathrm{OM}^{-1}$, переходящее при отрицательных напряжениях в резкий пик с максимумом при $V_{g}=-0.26$ В. Пик - это не физическая особенность, обусловленная слиянием в данной области полевых напряжений всех емкостных кривых, что приводит к ошибкам при расчетах — в формуле (1) фигурирует корень квадратный от разности емкостей - двух близких величин. Форма особенности (пик) связана и с резким замедлением уменьшения емкостей при переходе от -0.2 к $-0.5 \mathrm{~B}$ (рис. 1$)$.

Связь изгиба зон в полупроводнике $n$-типа со значением высокочастотной емкости $\bar{C}$ без учета эффектов размерного квантования и вырождения электронной системы на основе классических формул [9] может быть представлена в виде

$$
\frac{\bar{C}}{C_{i}-C}=\left(\frac{q V_{s h}}{T}\right)^{(1 / 2)}\left(e^{-v_{s}}+v_{s}-1\right)^{-(1 / 2)}\left|1-e^{-v_{s}}\right|,
$$

где $C_{i}$ - емкость диэлектрического слоя, $V_{s}-$ падение напряжения на полупроводнике, $v_{s}=-q V_{s} / T-$ безразмерный изгиб зон в полупроводнике $\left(v_{s}>0\right.$ при обеднении и $v_{s}<0$ при обогащении), $q$ - элементарный заряд, $T$ - температура в энергетических единицах, $V_{s h}=\frac{2 \pi \mathfrak{x}_{s} q N_{d} h^{2}}{\mathfrak{x}_{i}^{2}}, \mathfrak{x}_{s}$ и $\mathfrak{x}_{i}-$ диэлектрические проницаемости полупроводника и изолятора, $h$ - толщина окисла, $N_{d}$ - концентрация легирующей примеси у ГР. За величину $C_{i}$ принималось значение $\bar{C}$ для не поврежденного образца в состоянии глубокого обогащения 
Вычисление параметров $\left(V_{g},\left(\frac{q V_{s h}}{T}\right)^{(1 / 2)}\right.$ и $\left.N_{d}\right)$ при различных $v_{s}$

\begin{tabular}{c|c|c|c|c|c}
\hline$v_{s}$ & 0 & -1 & -2 & -3 & -4 \\
\hline$V_{g}, \mathrm{~B}$ & -0.199 & -0.225 & -0.258 & -0.284 & -0.312 \\
$\left(\frac{q V_{s h}}{T}\right)^{1 / 2}$ & $9.764 \cdot 10^{-2}$ & $1.011 \cdot 10^{-1}$ & $9.62 \cdot 10^{-2}$ & $9.744 \cdot 10^{-2}$ & $9.745 \cdot 10^{-2}$ \\
$N_{d}, \mathrm{~cm}^{-3}$ & $2.608 \cdot 10^{15}$ & $2.794 \cdot 10^{15}$ & $2.531 \cdot 10^{15}$ & $2.597 \cdot 10^{15}$ & $2.597 \cdot 10^{15}$
\end{tabular}

полупроводника. Поскольку в процессах высокотемпературного окисления поверхности кремния и очистки получаемых структур концентрация легирующей примеси у ГР $\mathrm{Si}-\mathrm{SiO}_{2}$ может измениться по сравнению с ее значением в толще полупроводниковой подложки [13], то значение $N_{d}$, а точнее говоря, величину параметра $V_{s h}$ необходимо определять непосредственно из экспериментальных данных. Для опытов при комнатной температуре пограничные состояния перезаряжаются практически вплоть до состояния плоских зон полупроводника. Поэтому вычислять значение параметра $V_{s h}$ из измеренных зависимостей $\bar{C}\left(V_{g}\right)$ следует в области полевых напряжений, соответствующих слабому обогащению поверхности полупроводника свободными электронами, где пограничные состояния уже практически заполнены и не изменяют свой заряд с ростом полевого напряжения, а эффекты размерного квантования еще не проявляются. В этом диапазоне выполняется соотношение

$$
\left(\frac{C_{i}}{C_{i}-\bar{C}}\right)^{2} \frac{2}{\bar{C}} \frac{T d \bar{C}}{q d V_{g}}=\frac{\left(e^{-2 v_{s}}+2 v_{s} e^{-v_{s}}-1\right)}{\left(e^{-v_{s}}+v_{s}-1\right) \mid 1-e^{-v_{s} \mid}},
$$

причем левая часть выражения (3) является функцией только полевого напряжения, а правая зависит только от изгиба зон полупроводника. Задавая $v_{s}=0 ;-1 ;-2 ;-3 ;-4$ из соотношения (3), можно найти соответствующие им величины $V_{g}$. По зависимости $\bar{C}\left(V_{g}\right)$ при этих $V_{g}$ из формулы (2) вычисляется искомое значение параметра $\left(\frac{q V_{s h}}{T}\right)^{(1 / 2)}$. В таблице приведены его величины для указанных изгибов зон не поврежденного образца, а также соответствующие им значения полевого напряжения и концентрации легирующей примеси. В дальнейших расчетах были использованы средние величины $\left(\frac{q V_{s h}}{T}\right)^{(1 / 2)}=9.796 \cdot 10^{-2}, N_{d}=2.625 \cdot 10^{15} \mathrm{~cm}^{-3}$; разброс данных составил менее $1.7 \%$.

Зависимости безразмерного изгиба зон в полупроводнике $v_{s}$ и величины $p_{s q}\left(p_{s q}-\right.$ выраженный в см${ }^{-2}$ суммарный заряд пограничных состояний и дырок на ГР) от напряжения на полевом электроде показаны на рис. 3 и 4. Значения $p_{s q}$ вычислялись из выражения

$$
\begin{aligned}
& p_{s q}=-\frac{C_{i} T}{S q} \\
& \times\left[\frac{q V_{g}}{T}+v_{s}+2\left(\frac{q V_{s h}}{T}\right)^{(1 / 2)}\left(e^{-v_{s}}+v_{s}-1\right)^{(1 / 2)} S g n v_{s}\right],
\end{aligned}
$$

где

$$
\operatorname{Sgnv_{s}}= \begin{cases}1, & v_{s}>0 \\ -1, & v_{s}<0\end{cases}
$$

Проанализируем полученные результаты. Как видно из рис. 1 , стресс приводит к существенно более низким уровням выхода емкостей образца на плато при больших положительных полевых напряжениях. Это свидетельствует о резком увеличении концентрации поверхностных состояний. Их заполнение электронами происходит при пиннинге уровня Ферми, что означает примерно постоянный изгиб зон в полупроводнике, и поэтому на этих плато $\bar{C}$ (рис. 2 ) существенно меньше $C_{i}$. Графики на рис. 3 и 4 подтверждают данный вывод: на плато $v_{s} \approx-3.6$, а число отрицательных зарядов доходит до $p_{s q}=8 \cdot 10^{12} \mathrm{~cm}^{-2}$. Отсюда следует, что уровень энергии электрона на данных состояниях лежит ниже дна зоны проводимости примерно на $\left[T \ln \left(N_{c} / N_{d}\right)-3.6\right]^{1}$ или на 0.14 эВ. Кривая $p_{s q}\left(V_{g}\right)$ в неповрежденном образце (рис. 4) частично изображена пунктирной кривой. Это указание на нефизический характер данной зависимости в области больших полевых напряжений: величина $p_{s q}$ должна быть постоянна, а она растет с $V_{g}$. При таких обогащающих изгибах зон в полупроводнике уже существенны эффекты размерного квантования и вырождения электронной системы. Поэтому использование в расчетах выражения (2), основанного на классических представлениях и статистике Больцмана, приводит в этой области $V_{g}$ к неправильным значениям рассчитываемых величин. В подтверждение результатов работы [8], у нас также наблюдается повышение темпа генерации неосновных носителей заряда после полевого воздействия. Рождение дырок происходит в основном на обратных ветвях измерений, и оценивать величину их накопления можно по расхождению кривых $p_{s q}$. Но при этом сравнение величин $p_{s q}$ нужно вести для одинаковых изгибов зон полупроводника. Поэтому на вставке к рис. 4 представлены зависимости $p_{s q}\left(v_{s}\right)$ для прямой и обратной ветвей измерений в состояниях до стресса и сразу после стресса изолятора. Видно, что требуемые значения концентрации неосновных носителей заряда достигаются раньше в состоянии сразу после стресса.

В заключение сформулируем основной вывод данной работы. Воздействие на сверхтонкий изолирующий слой сильного, но допробойного, электрического поля приводит к образованию дополнительных пограничных локализованных электронных состояний с уровнем, отстоящим от дна зоны проводимости кремния на 0.14 эВ. Поскольку перезарядка вновь образованных центров с ростом полевого напряжения заведомо обеспечивает

${ }^{1} N_{c}-$ эффективная плотность состояний в зоне проводимости кремния. 


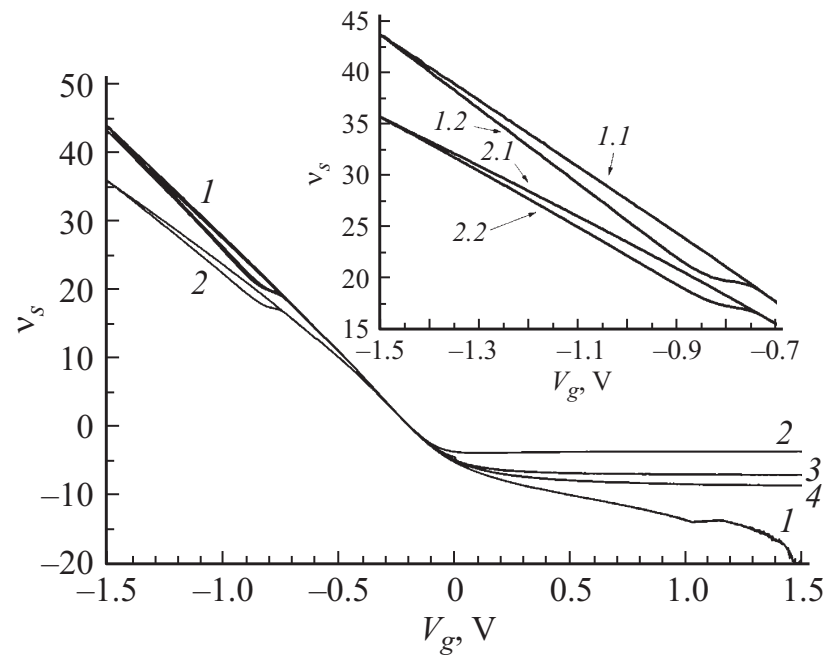

Рис. 3. Зависимости безразмерного изгиба зон в полупроводнике от напряжения на полевом электроде: 1 - до стресса, 2 - сразу после стресса, 3 - через 2 ч после стресса, 4 - через 4 ч после стресса. При отрицательных $V_{g}$ кривые 3 и 4 практически сливаются с 1 . На вставке: 1.1 - до стресса (прямая ветвь), 1.2 - до стресса (обратная ветвь), 2.1 - сразу после стресса (прямая ветвь), 2.2 - сразу после стресса (обратная ветвь).

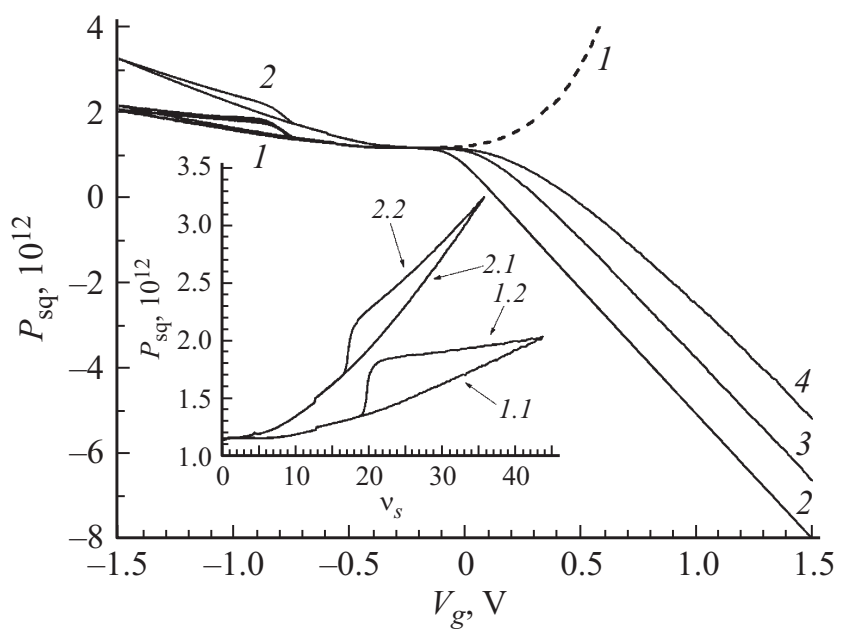

Рис. 4. Зависимость суммарного заряда пограничных состояний и дырок от напряжения на полевом электроде: 1 - до стресса, 2 - сразу после стресса, 3 - через 2 ч после стресса, 4 - через 4 ч после стресса. При отрицательных $V_{g}$ кривые 3 и 4 практически сливаются с 1. На вставке: 1.1 - до стресса (прямая ветвь), 1.2 - до стресса (обратная ветвь), 2.1 - сразу после стресса (прямая ветвь), 2.2 - сразу после стресса (обратная ветвь).

накопление у ГР кремний-окисел избыточного заряда с концентрацией $8 \cdot 10^{12} \mathrm{~cm}^{-2}$, то общее количество новых локализованных состояний может превышать $10^{13} \mathrm{~cm}^{-2}$. Такое большое значение заряда у ГР полупроводник-диэлектрик должно оказать решающее влияние на изменение туннельной вольт-амперной характеристики $\mathrm{Si}-\mathrm{MOП} \mathrm{структур} \mathrm{после} \mathrm{стресса.}$
Работа выполнена при частичной финансовой поддержке Программы фундаментальных исследований президиума РАН „Наноструктуры: физика, химия, биология, основы технологий“ и иранта РФФИ № 16-07-00666.

\section{Список литературы}

[1] G. Cellere, S. Gerardin, Al. Paccagnella. In: Defects in Microelectronic Materials and Devices, ed. by D.M. Fleetwood, S.T. Pantelides, R.D. Schrimpf (CRC Press, 2008) chap. 17, p. 497.

[2] E.H. Poindexter. Semicond. Sci. Technol., 4, 961 (1989).

[3] F.B. McLean. IEEE Trans. Nucl. Sci., 27, 1651 (1980).

[4] T.R. Oldham, F.B. McLean, H.E. Boesch, J.M. McCarrity. Semicond. Sci. Technol., 4, 986 (1989).

[5] M.L. Reed. Semicond. Sci. Technol., 4, 980 (1989).

[6] M. Durr, Z. Hu, A. Biedermann, U. Hofer, T.F. Heinz. Phys. Rev. B, 63, 121315(R) (2001).

[7] K. Komiya, Y. Omura. J. Appl. Phys., 92, 2593 (2002).

[8] Е.И. Гольдман, Н.Ф. Кухарская, В.Г. Нарышкина, Г.В. Чучева ФТП, 45, 974 (2011).

[9] S.M. Sze, K.Ng. Kwok. Physics of semiconductor devices (N.J., John Willey and Sons, Inc., 2007).

[10] L.F. Lonnum, J.S. Johannessen. Electron. Lett., 22, 456 (1986).

[11] Е.И. Гольдман, А.И. Левашова, С.А. Левашов, Г.В. Чучева. ФТП, 49, 483 (2015).

[12] J.Y. Kevin, H. Chenming. IEEE Trans. Electron. Dev., 46, 1500 (1999).

[13] E.H. Nicollian, I.R. Brews. MOS (Metal Oxide Semiconductor) Physics and Technology (N.Y., John Willey and Sons, 1982).

Редактор Г.А. Оганесян

\section{The generation of surface electronic states at the silicon-ultrathin oxide interface during a field stress of metal-oxide-semiconductor structures}

\section{E.I. Goldman, S.A. Levashov, V.G. Naryshkina, G.V. Chucheva}

Fryazino branch of the Kotel'nikov Institute of Radioengineering and Electronics of Russian Academy of Sciences, 141190 Fryazino, Russia

Abstract High-frequency capacitance-voltage $(C-V)$ characteristics of metal-oxide-semiconductor (MOS) structures on $n$-Si-substrates with the oxide thickness of $39 \AA$, subjected to damage in the field stress, are measured. It is shown, that the exposure to an ultra-thin insulating layer of the strong, but before breakdown, electric field leads to the formation of a large number of additional interface localized electronic states at a level, spaced from the bottom of the conduction band of the silicon to $0.14 \mathrm{eV}$. It has been found, that the recharge of newly formed centers with increasing of field voltage provides the accumulation at the silicon-oxide interface of the excess charge up to $8 \cdot 10^{12} \mathrm{~cm}^{-2}$. The lifetime, born in field stress of local centers, is of two days; then depending of localized charge at the semiconductor-dielectric interface on the gate voltage in states before and after the stress are practically the same. 